\title{
Traumatic Brain Damage: Severe Brain Damage: Coma and Disorders of Consciousness
}

\author{
Steven Laureys
}

\section{Contents}

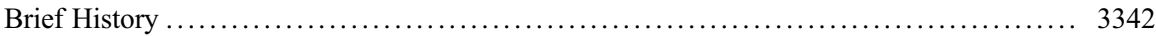

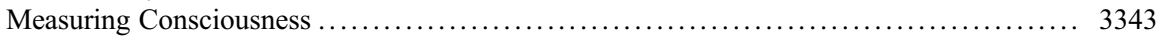

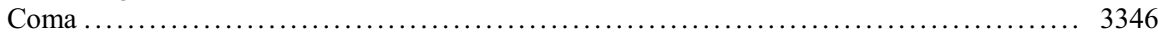

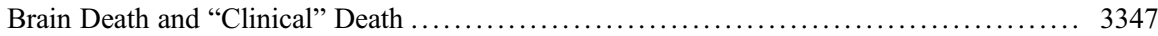

"Near" Death ........................................................... 3349

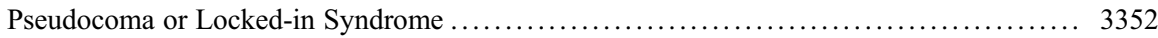

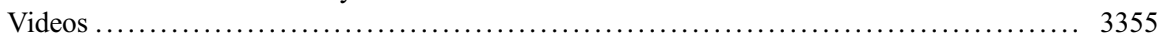

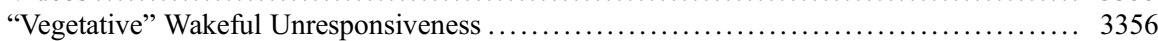

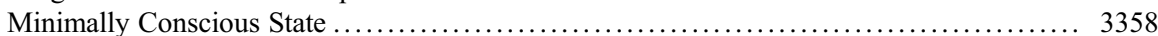

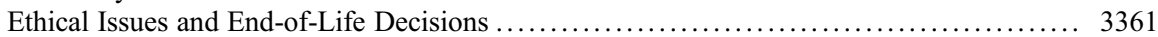

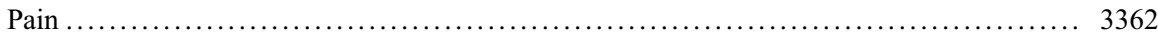

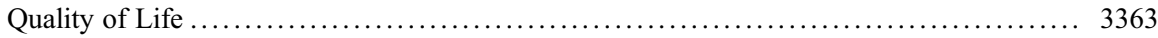

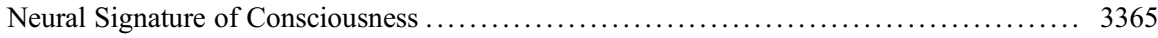

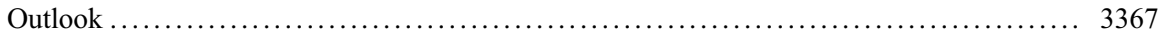



\section{Abstract}

Progress in intensive care efforts has increased the number of patients who survive severe acute brain damage. Although the majority of these patients recover from coma within the first days after the insult, some permanently lose all brain function (i.e., brain death), while others evolve to a state of "vegetative" unresponsive wakefulness. Those who recover typically progress through different stages before fully or partially recovering consciousness (minimally conscious state; MCS). Functional neuroimaging and electrophysiology studies are changing our understanding of patients with coma and related states. Some

\footnotetext{
S. Laureys $(\bowtie)$

Cyclotron Research Center and Neurology Department, University of Liège Coma Science Group, Liège, Belgium

e-mail: steven.laureys@ulg.ac.be
} 
severely brain-damaged patients may show residual cortical processing in the absence of behavioral signs of consciousness. When "vegetative"/unresponsive patients show minimal signs of consciousness but are unable to reliably communicate, the term "minimally responsive" or "minimally conscious state" (MCS) is used.

\section{Keywords}

Active cognitive event-related potentials - Apallic syndrome $\cdot$ Brain death $\bullet$ Clinical death $\cdot$ Coma $\bullet$ Consciousness $\bullet$ Cortical death $\bullet$ Decerebrate $\bullet$ Decorticate $\bullet$ External awareness $\bullet$ Glasgow coma scale $\bullet$ Greyson scale $\bullet$ Hemodynamic functional neuroimaging studies - Hollow-skull sign - Internal awareness $\bullet$ Locked-in syndrome $\cdot$ Minimally conscious state $\cdot$ Near-death experiences $\bullet$ Organic hypotheses $\cdot$ Pain - Positron emission tomography (PET) scanning • Pseudocoma $\bullet$ Quality of life $\bullet$ Reticular activating system $\bullet$ Vegetative wakeful unresponsiveness

\section{Brief History}

Videos from the author can be found here: www.comascience.org.

Progress in intensive care efforts has increased the number of patients who survive severe acute brain damage. Although the majority of these patients recover from coma within the first days after the insult, some permanently lose all brain function (i.e., brain death), while others evolve to a state of "vegetative" unresponsive wakefulness. Those who recover typically progress through different stages before fully or partially recovering consciousness (minimally conscious state; MCS). Functional neuroimaging and electrophysiology studies are changing our understanding of patients with coma and related states. Some severely braindamaged patients may show residual cortical processing in the absence of behavioral signs of consciousness. When "vegetative"/unresponsive patients show minimal signs of consciousness but are unable to reliably communicate, the term "minimally responsive" or "minimally conscious state" (MCS) is used. Recently, a subcategorization was proposed based on the complexity of the observed behaviors: MCS+ describes high-level responses (e.g., command following), and MCSis used when "nonreflex" low-level responses are encountered (e.g., visual pursuit, localization of noxious stimuli, or contingent behavior to emotional stimuli). Finally, patients who show nonmotor-dependent evidence of consciousness or communication only assessable via paraclinical testing (e.g., functional MRI or evoked potentials) can be considered to be in a "functional locked-in syndrome." An improved assessment of brain function in coma and related states not only is changing nosology (Fig. 1) but also offers a better-documented diagnosis and prognosis and helps to further identify the neural correlates of human consciousness. 


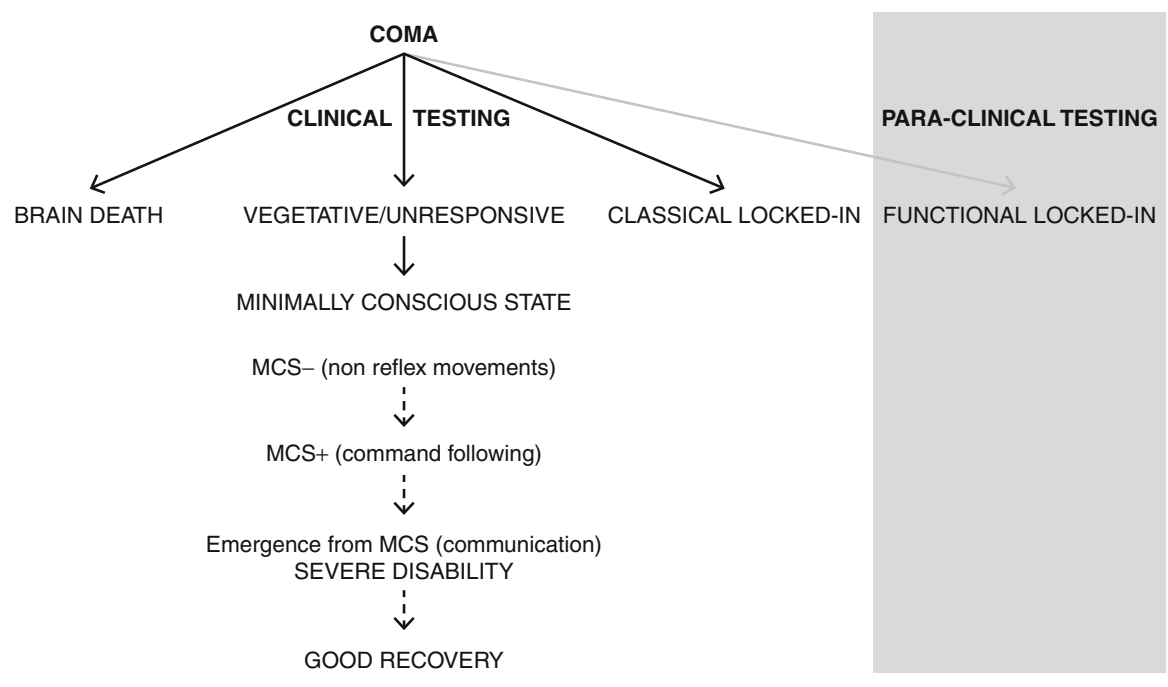

Fig. 1 Proposed nosology of the diagnostic entities that can be encountered following coma, based on clinical behavioral evaluation (in white, inferred from assessing motor responses) and based on novel paraclinical functional neuroimaging studies (in gray, inferred from assessing brain responses) - see text for details

\section{Measuring Consciousness}

There is at present no satisfactory, universally accepted definition of consciousness. For the purposes of clinical neurosciences, consciousness consists of two basis components: wakefulness (i.e., arousal or vigilance) and awareness (i.e., content of consciousness). Awareness in turn can be divided into external awareness (i.e., sensory or perceptual awareness of the environment) and internal awareness (i.e., stimulus-independent thoughts, mental imagery, inner speech, daydreaming, or mind wandering). Figure 2 shows that in normal physiological states, level of arousal and content of consciousness (environmental and self) are positively correlated. You need to be awake in order to be aware (rapid eye movement or REM sleep and lucid dreaming being notorious exceptions). Patients in pathological or pharmacological coma (i.e., general anesthesia) are unconscious because they cannot be awakened. The "vegetative" state is a dissociated state of consciousness (i.e., patients being seemingly awake but lacking any behavioral evidence of nonreflex behavior or command following). Other examples of dissociation between the presence of reflex movements in the absence of command following can be encountered in sleepwalking and in some form of epilepsy (absence and complex partial seizures).

Bedside evaluation of residual brain function in severely brain-damaged patients is difficult because motor responses may be very limited or inconsistent. In addition, consciousness is not an all-or-none phenomenon, and its clinical assessment relies 


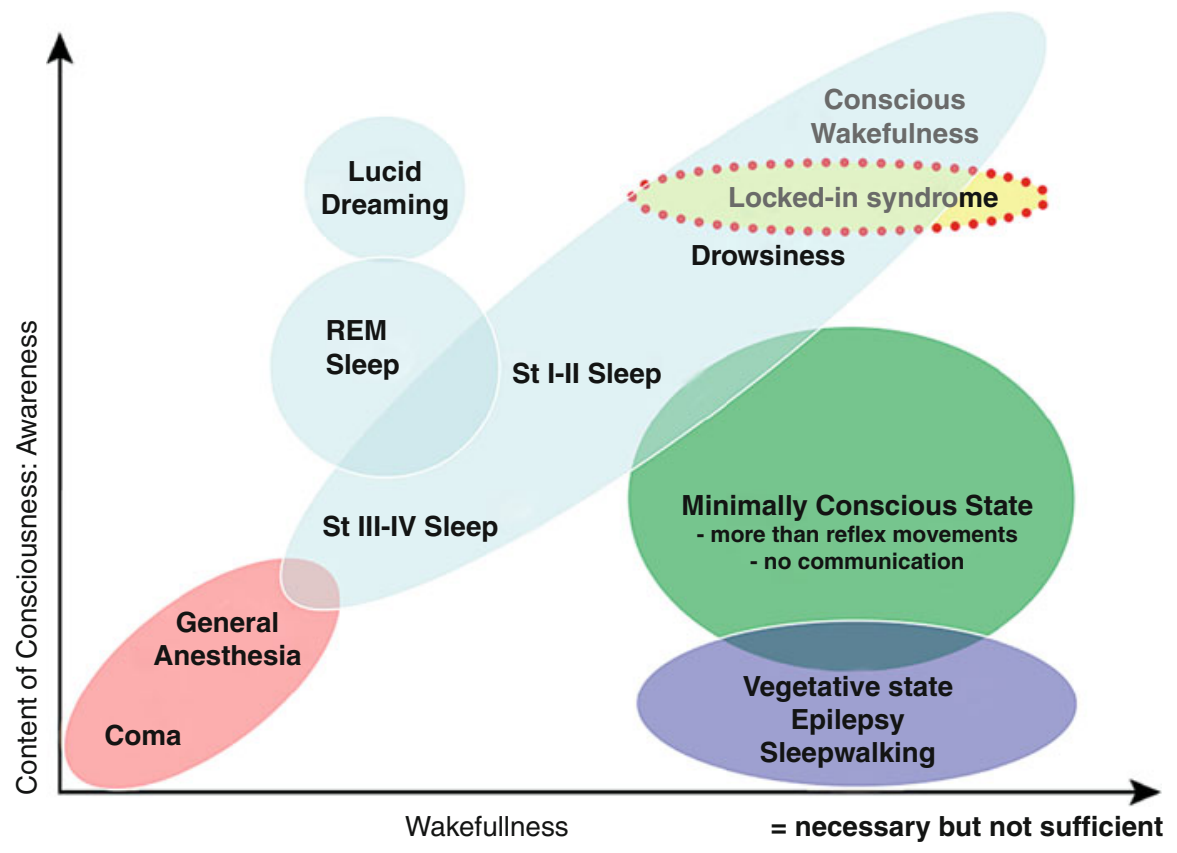

Fig. 2 Wakefulness or arousal positively correlates with the content of consciousness (i.e., awareness), as illustrated in the case of normal physiological modifications of consciousness during sleep (with the exception of the oneiric activity during REM sleep). Patients in pathological or pharmacological coma (i.e., general anesthesia) are unconscious because they cannot be awakened. The "vegetative" state illustrates the dissociation between wakefulness and awareness. In the locked-in syndrome, patients are unable to move and express their awareness except via eye-coded communication

on inferences made from observed responses to external stimuli at the time of the examination. How do we quantify consciousness at the bedside? Clinically, arousal will simply be assessed by examining the presence of spontaneous or stimulusinduced eye opening, and the presence of reproducible command following will be taken as proof of (external) awareness. Additionally, the presence of spontaneous or induced nonreflex behaviors will be considered as evidence of (minimal) consciousness. The bedside examination of consciousness in severe brain-damaged patients often is very challenging because observed movements may be very small, inconsistent, and easily exhausted, potentially leading to diagnostic errors.

The "Glasgow Coma Scale" is the most widely used standardized bedside tool to quantify wakefulness and awareness in coma. It was devised as a formal scheme to overcome the ambiguities that arose when information about comatose patients was presented and groups of patients compared. The scale has three components: eye, verbal, and motor response to external stimuli (Fig. 3). The best or highest responses are recorded. The presence of spontaneous eye opening indicates that the arousal mechanisms of the brain stem are active. The presence of verbal responses indicates the restoration of a high degree of interaction with the environment (i.e., awareness). 


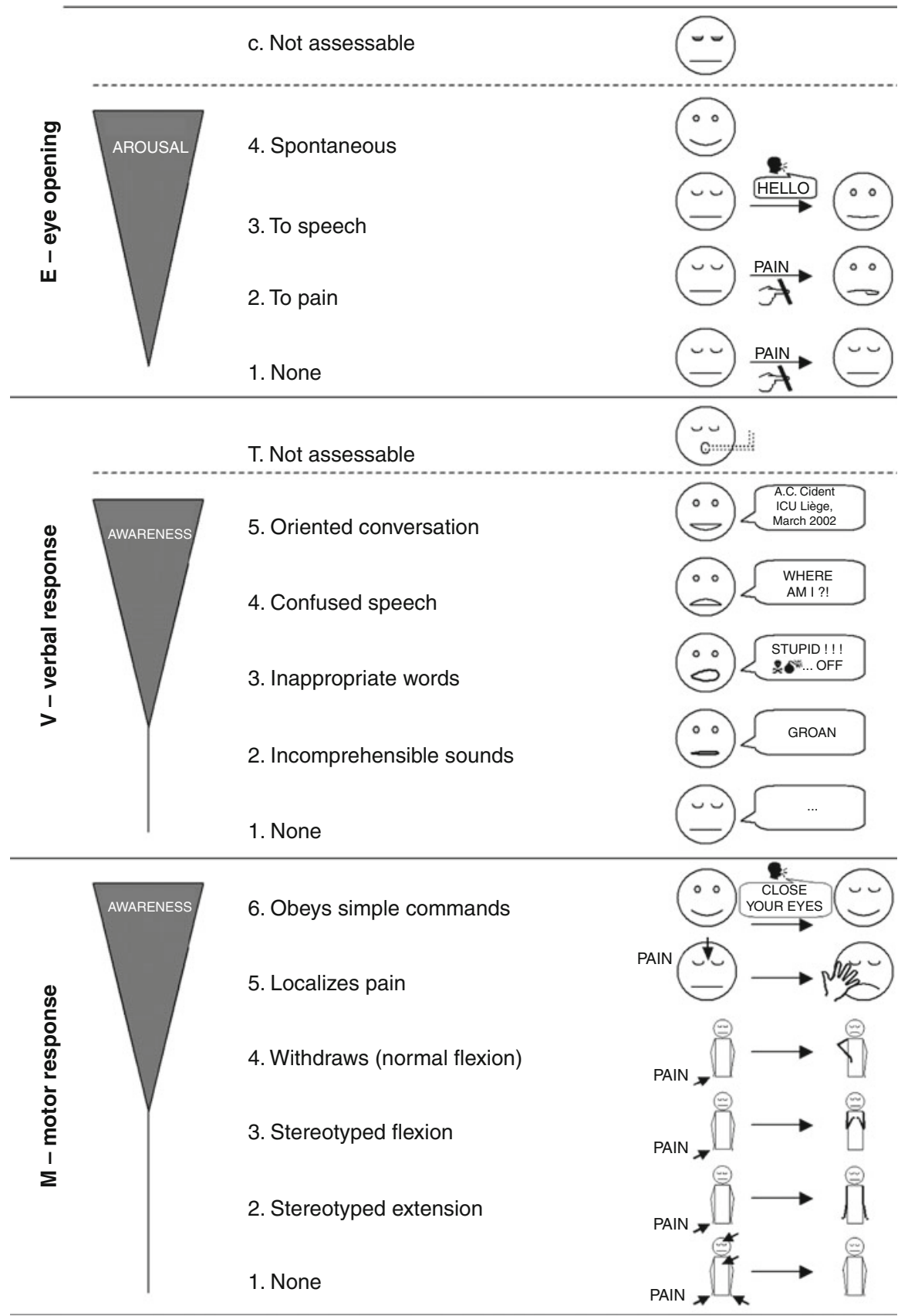

Fig. 3 The glasgow coma scale 
The motor response first assesses whether the patient obeys to simple commands, given in verbal, gestural, or written form. If there is no response, a painful stimulus is applied. Stereotyped flexion responses are the most common of the motor reactions observed in severely brain-injured patients; they are also the most enduring. Extensor posturing is more easily distinguished and is usually associated with adduction, internal rotation of the shoulder, and pronation of the forearm. The term "decerebrate" and "decorticate" rigidity should be avoided because it implies a specific physioanatomical correlation, known to be not necessarily correct. It is tempting to sum the three components of the Glasgow Coma Scale into a total score, ranging from 3 to 15 . However, given the increased use of intubation, ventilation, and sedation of patients with impaired consciousness before arrival at hospital, patients might wrongly being scored as GCS 3/15 rather than being more appropriately reported as impossible to assess or score. One of the most frequently expressed reservations regarding the scale has been its failure to incorporate brain stem reflexes. Many coma scales that include brain stem indicators have been proposed (e.g., the Glasgow Liège Scale and more recently the Full Outline of Unresponsiveness), but none are as extensively used as the Glasgow Coma Scale.

After the acute setting, when patients have emerged from their coma and the difference needs to be made between "vegetative" or minimally conscious patients, Glasgow Coma Scale assessment should be replaced by other scales. For chronic disorders of consciousness, the most validated scale is the Coma Recovery ScaleRevised. This scale assesses auditory, visual, verbal, and motor functions as well as communication and arousal level (the total score ranges between 0 and 23). It has shown superior performance in detecting minimal signs of consciousness after the period of coma, as compared to other scales or unstructured assessment. A recent study showed that the clinical consensus diagnosis could be incorrect in more than $40 \%$ of patients considered to be "vegetative" if not assessed by Coma Recovery Scale assessment. As we will see, locked-in syndrome patients may also be mistakenly considered unconscious. The problem of assessing consciousness in severe brain damage may be further complicated when patients have underlying deficits in the domain of verbal or nonverbal communication functions, such as aphasia, agnosia, or apraxia.

\section{Coma}

Coma is characterized by the absence of arousal and thus also of awareness. It is a state of unarousable unresponsiveness in which the patient lies with the eyes closed and shows no signs of awareness. The patient lacks the spontaneous periods of wakefulness and eye opening induced by stimulation that can be observed in the "vegetative" state. The most frequent causes of coma are traumatic or ischemic brain damage. It can result from diffuse bihemispheric cortical (e.g., after cardiac arrest) or white matter damage secondary to diffuse neuronal or axonal injury (e.g., after deceleration traffic accidents) or from focal but extensive brain stem lesions (e.g., after stroke or hemorrhage). To be clearly distinguished from syncope, concussion, or other states of transient unconsciousness, coma must persist for at least $1 \mathrm{~h}$. 
Fig. 4 Overall cortical metabolism in coma, "vegetative," minimally conscious, and locked-in syndromes, as compared to slow wave sleep and anesthesia

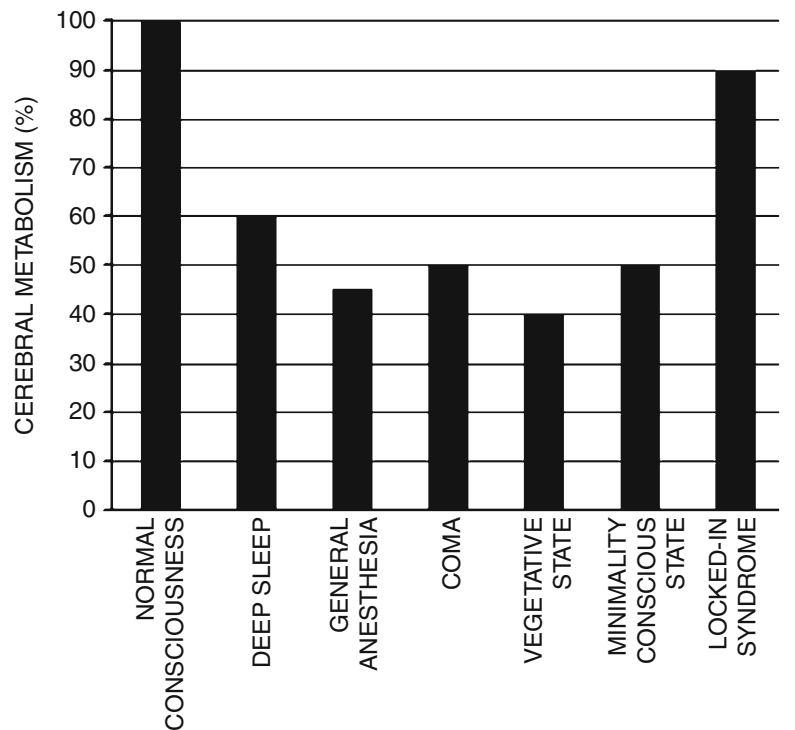

In general, comatose patients who survive begin to awaken and recover gradually within 2-4 weeks. This recovery may go no further than wakeful unresponsiveness or minimal responsiveness, or these may be stages (brief or prolonged) on the way to more complete recovery of consciousness. The prognosis of coma survivors following brain anoxia is worse than following trauma. In anoxic coma, absent or stereotyped motor responses and absent pupillary reflexes indicate bad outcome. Paraclinically, isoelectrical ("flat") or "burst-suppression" EEG and the bilateral absence of somatosensory evoked potentials in primary cortex (called N20 wave or potential) are strong indicators of bad outcome. In contrast, auditory oddball evoked potentials showing an intact mismatch negativity effect forebodes an outcome better than death or unresponsive wakefulness.

PET studies show that, on average, gray matter metabolism is $50-70 \%$ of normal values in comatose patients of traumatic or hypoxic origin. A global depression of cerebral metabolism is not unique to coma. When different anesthetics are titrated to the point of unresponsiveness, the resulting reduction in brain metabolism is similar as that observed in comatose patients. Another example of transient metabolic depression can be observed during deep sleep (stages III and IV). In this daily physiological condition, cortical cerebral metabolism can drop to nearly $40 \%$ of normal values (Fig. 4).

\section{Brain Death and "Clinical" Death}

The widespread use of the artificial respirator in the 1960s led to the redefinition of death based on neurological criteria (i.e., brain death or irreversible coma with absent brain stem reflexes). Brain death means human death determined by neurological criteria. It is an unfortunate term as it misleadingly suggests that there are two types of death: brain death and "regular" death. There is, however, only one type of death which can be 
measured in two ways: by cardiorespiratory and by neurological criteria. The currently most accepted definition of death is the permanent cessation of the critical functions (i.e., respiration, circulation, and consciousness) of the organism as a whole.

Brain death results from irreversible loss of brain and brain stem function and is classically caused by a brain lesion (e.g., massive traumatic injury, intracranial hemorrhage, or anoxia) which increases intracranial pressure to values superior to mean arterial blood pressure and hence causes intracranial circulation to cease and damage the brain stem due to herniation. Anatomopathology in brain death patients receiving maximal artificial means of support will inevitably end up showing the so-called respirator brain: surface vasocongestion due to venous engorgement, thrombosis in cortical veins and sinuses, subarachnoid hemorrhage, and cortical congestion and hemorrhage will be observed after about $12 \mathrm{~h}$ of a nonperfused state. After about a week, an autolyzed liquefied brain will pour from the opened skull.

The concept of brain death as defining the death of the individual is largely accepted. Most countries have published recommendations for the diagnosis of brain death, but the diagnostic criteria differ from country to country. Some rely on the death of the brain stem only; others require death of the whole brain including the brain stem. However, the clinical assessments for brain death are very uniform and based on the irreversible loss of all brain stem reflexes and the demonstration of continuing apnea in a persistently comatose patient. Table 1 shows the clinical criteria for brain death, as defined by the American Academy of Neurology - including the very important apnea testing - which have been used to model many institutional policies. Since the first definition of the neurologic criteria of death over 45 years ago, no patient in apneic coma properly declared brain (or brain stem) death has ever regained consciousness.

It is important to stress that clinical death is a term to be avoided, referring in popular media to cessation of blood circulation and breathing. Under the US Uniform Determination of Death Act, a person is dead when physicians determine, by applying prevailing clinical criteria, that cardiorespiratory or brain functions are absent and cannot be retrieved. Without resuscitation, recovery of brain function after more than $3 \mathrm{~min}$ after a cardiac arrest is rare. This notion of irreversibility is reflected in the "Pittsburgh Protocol" for organ "donation after cardiac death." Here, patients who are hopelessly brain damaged (but not brain dead) can have their

Table 1 Diagnostic criteria for brain death, as published by the American Academy of Neurology

Demonstration of coma

Evidence for the cause of coma

Absence of confounding factors, including hypothermia, drugs, electrolyte, and endocrine disturbances

\begin{tabular}{l}
\hline Absence of brain stem reflexes \\
\hline Absent motor responses \\
\hline Apnea \\
\hline A repeat evaluation in $6 \mathrm{~h}$ is advised, but the time period is considered arbitrary \\
Confirmatory laboratory tests are only required when specific components of the clinical testing \\
cannot be reliably evaluated
\end{tabular}




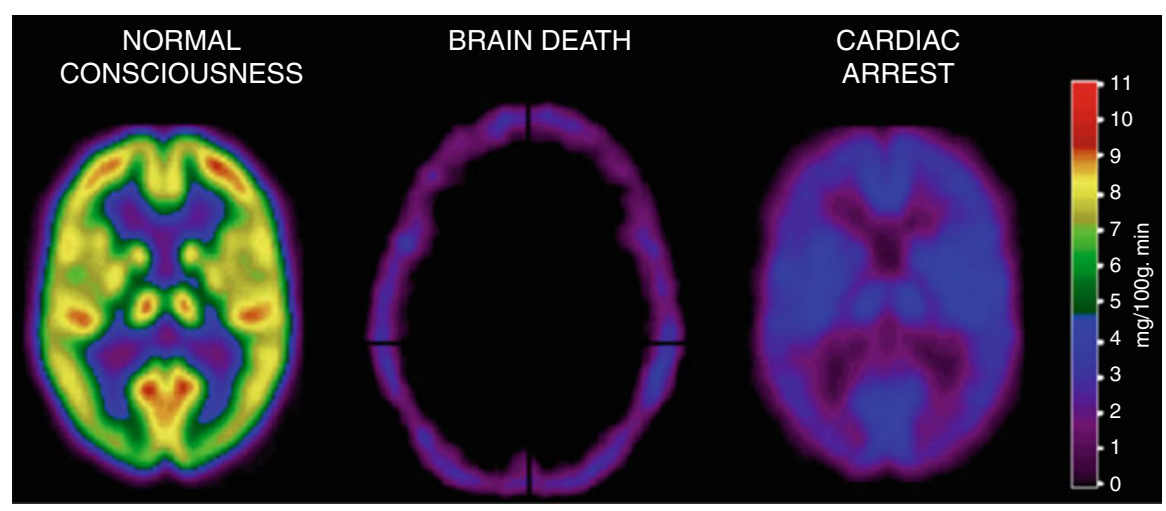

Fig. 5 Positron emission tomography (PET) scans illustrating the "empty skull sign" in brain death and massive global decrease of brain metabolism in a cardiac arrest survivor. The color scale shows the amount of glucose metabolized per $100 \mathrm{~g}$ of brain tissue per minute

life-sustaining therapy (i.e., positive-pressure ventilation) withdrawn. Once their heart stops beating for a period of 3-10 min (that varies by protocol), they can be declared dead (and only then can be organs procured).

Defining death and organ harvesting are inextricably linked because of the "dead donor rule." This rule requires patients to be declared dead before the removal of lifesustaining organs for transplantation. Some authors have proposed that death be defined by the permanent cessation of the higher functions of the nervous system that demarcate man from the lower primates. This neocortical or higher brain death definition has been mainly developed by philosophers, and its conceptual basis rests on the premise that consciousness, cognition, and social interaction, not the bodily physiological integrity, are the essential characteristics of human life. Based on this definition, vegetative patients following an acute injury or chronic degenerative disease and anencephalic infants are considered dead. The neocortical definition of death has never convinced medical associations or courts.

Cerebral angiography and transcranial Doppler sonography can be used with very high sensitivity and $100 \%$ specificity to document the absence of cerebral blood flow in brain death. Similarly, radionuclide cerebral imaging such as single photon emission $\mathrm{CT}$ and PET classically show the so-called hollow-skull sign confirming the absence of neuronal function in the whole brain (see Fig. 5). The EEG in brain death shows absent electrocortical activity (i.e., isoelectric or "flat" recording) with a sensitivity and specificity of $90 \%$. It is the most validated and, because of its wide availability, preferred confirmatory test for brain death implemented in many countries' guidelines.

\section{“Near" Death}

Near-death experiences and out-of-body experiences remain to be fascinating phenomena, which are abundant in popular beliefs, mythology, and spiritual experiences of many ancient and modern societies. At present, there exists no universally 
accepted definition of near-death experiences. Given that, there are many ways of categorizing its phenomenological elements. Common elements are ineffability, seeing a dark tunnel, experiencing a bright light as a "being of light," meeting "spiritual beings," being "out of the body," coming back "into the body," feelings of peace and quiet, panoramic life review, experiencing a realm in which all knowledge exists, and sensing a border or limit.

Clinical studies suggest that characteristics of near-death experiences are a culturally invariant physiological reality that can be investigated scientifically. The scale most commonly used to quantify the subjective experience of near-death experiences is the "Greyson Scale," consisting of 146 questions. In order to consider the subjective report as being a true near-death experience, a minimum score of 7 on 32 needs to be obtained. What causes near-death experiences? They seem to occur in a minority of individuals close to death (after both traumatic and nontraumatic coma) or in situations of intense physical or emotional danger. Most studies have been performed on cardiac arrest survivors. Spiritual interpretations consider their existence as evidence that the mind (i.e., spirit or soul) can be separated from the physical body. Supporters of this theory consider that near-death experiences provide a glimpse of the spiritual realm to which the soul migrates after death. The second category encompasses psychological explanations according to which near-death experiences are a type of depersonalization acting as a protection against the threat of death in situations of intense danger, by allowing an engagement in pleasurable fantasies. Others proposed a concept of psychological absorption, which may be defined as the tendency to focus attention on imaginative or selected sensory experiences to the exclusion of stimuli from the external environment. We will here focus on the so-called organic hypotheses, accounting for components of near-death experiences in terms of brain dysfunctions and alterations.

Prospective studies of cardiac arrest patients show that about $10 \%$ report memories or near-death experiences of their period of "coma" or unconsciousness. Most patients reported these to be pleasant. Due to the small number of patients studied, it is not possible to draw any clear conclusions regarding possible causative physiological factors such as administered drugs, duration of cardiac arrest or dysrhythmia, hypoxia, hypercarbia, or electrolyte disturbances around the period of arrest. Younger people and women seem more likely to report a near-death experience. These experiences often produce long-lasting effects in terms of increase in belief in an afterlife and decreased fear of death, increase interest in the meaning of one's own life, and increased social awareness such as showing love and accepting others.

Anoxic or traumatic brain damage leads to a complex neurometabolic cascade with the release of multiple neurotransmitters which, combined with administered centrally acting drugs during resuscitation or intensive care, may produce such effects as analgesia, euphoria, and detachment. These effects combined with regional hypoxic neuronal dysfunction may produce epileptiform discharges, possibly leading to the reported phenomenology of near-death experiences. There exists no EEG data about brain function in the critical clinical period that is assumed to be associated with near-death experiences. Indeed, in all peer-reviewed studies, loss of consciousness was "diagnosed" only by electrocardiogram examinations, independently of neurological records. 
One of the principal components of near-death experiences is the out-of-body experience, defined by the presence of (1) disembodiment (i.e., location of the self outside one's body), (2) the impression of seeing the world from an elevated and distanced visuospatial perspective, and (3) the impression of seeing one's own body from this perspective (i.e., autoscopy). There is increasing evidence showing that out-of-body experiences may result from a deficient multisensory integration at the right temporoparietal junction area. In two patients, focal electrical stimulation of this area induced repeated out-of-body experiences (Fig. 6). In studies of neurological patients suffering from epilepsy-related "out-of-body experiences,"

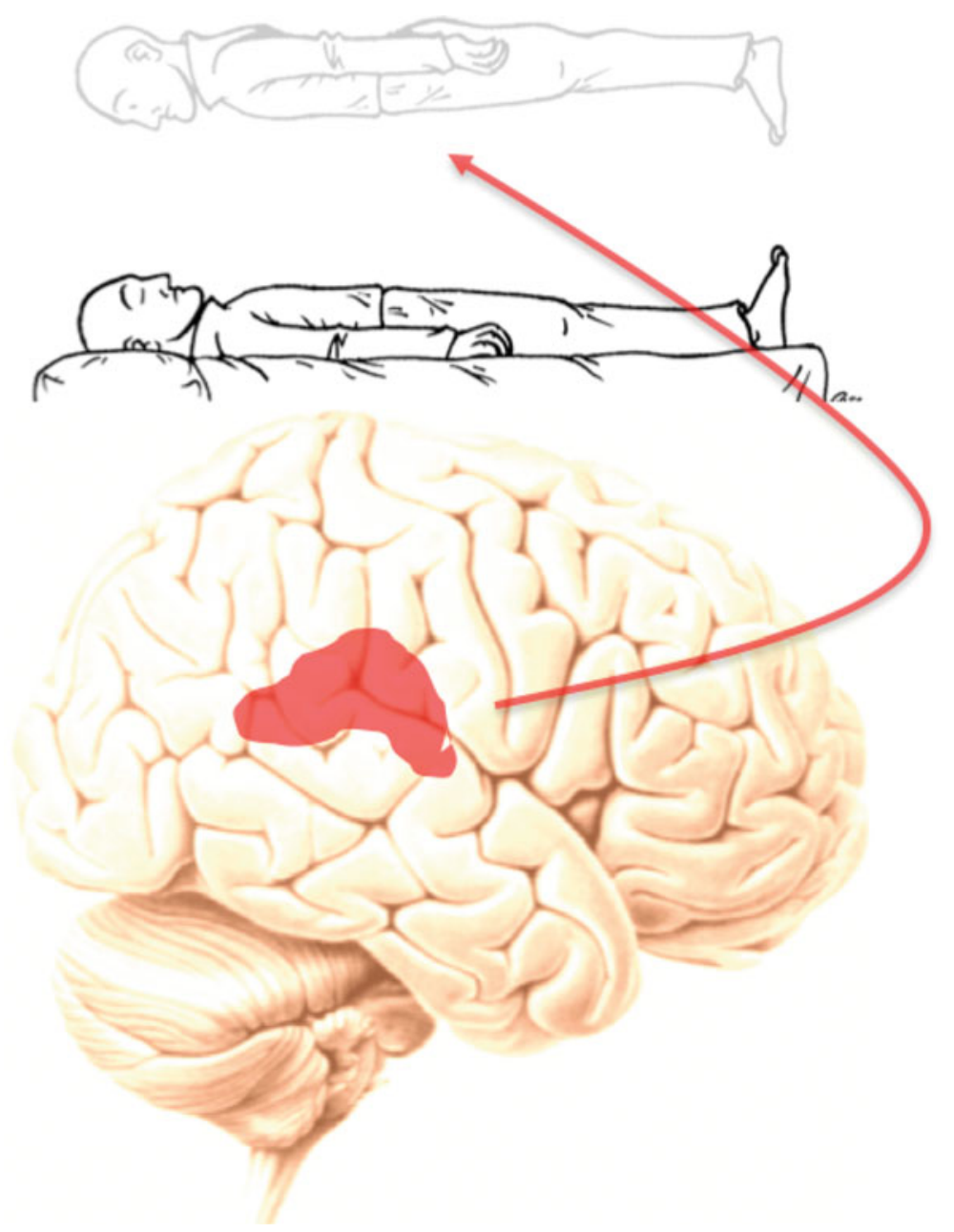

Fig. 6 One of the principal components of the near-death experience is the out-of-body experience. Studies have shown that electrical stimulation of the right temporoparietal junction (highlighted in red) could repeatedly generate these experiences in controlled settings 
lesion overlap was centered on the right temporoparietal junction. Altered spatial self-recognition seems mediated by this area that is known to be involved in vestibular-somatosensory integration of body orientation in space.

Similarly, based on knowledge from functional neuroimaging and clinical neurological studies, it can be hypothesized that the other distinctive dimensions of neardeath experiences can be related to particular regional brain dysfunction: (1) occipital and optic radiation area lesions could account for tunnel and light vision; (2) mesiotemporal areas (encompassing amygdala and hippocampus) for life review, memory flashback, and enhanced emotions; (3) left temporoparietal junction areas for the meeting of spirits; (4) anterior cingulate areas for the state of painlessness and well-being; (5) corticostriatal networks for time distortion; and (6) biparietal posterior cortices for transcendental oneness, cosmic unity, and mystical experiences. But further studies are awaited to better document the functional neuroanatomy of the complex subjectivity of near-death experiences.

\section{Pseudocoma or Locked-in Syndrome}

The locked-in syndrome describes patients who are awake and conscious but have no means of producing speech and limb or face movements (Table 2). The syndrome can be categorized on the basis of the extent of motor impairment: (a) classical locked-in syndrome is characterized by total immobility except for vertical eye movements or blinking; (b) incomplete locked-in syndrome permits remnants of voluntary motion such as moving a finger or the head; and (c) total locked-in syndrome consists of complete immobility including all eye movements combined with preserved consciousness. The cause is most frequently a ventral pontine lesion of the brain stem due to stroke or hemorrhage (but traumatic and other causes can also be encountered) (Fig. 7).

The term was first introduced to medicine in the 1960s, but the earliest example of a "locked-in" patient comes from Alexandre Dumas's novel The Count of Monte Cristo (1844). Herein a character, Monsieur Noirtier de Villefort, was depicted as "a corpse with living eyes." For more than 6 years, a helper pointed at words in a dictionary and Mr. Noirtier indicated with his eyes the words he wanted. Some years later, Emile Zola wrote in his novel Thérèse Raquin (1868) about a paralyzed woman who "was buried alive in a dead body" and "had language only in her eyes." Dumas and Zola highlighted the locked-in condition before the medical community did. In 1995, Frenchmen

Table 2 Diagnostic criteria for locked-in syndrome, as published by the American Congress of Rehabilitation Medicine

The presence of sustained eye opening (bilateral ptosis should be ruled out as a complicating factor)

Preserved basic cognitive abilities

Aphonia or severe hypophonia

Quadriplegia or quadriparesis

A primary mode of communication that uses vertical or lateral eye movement or blinking of the upper eyelid 


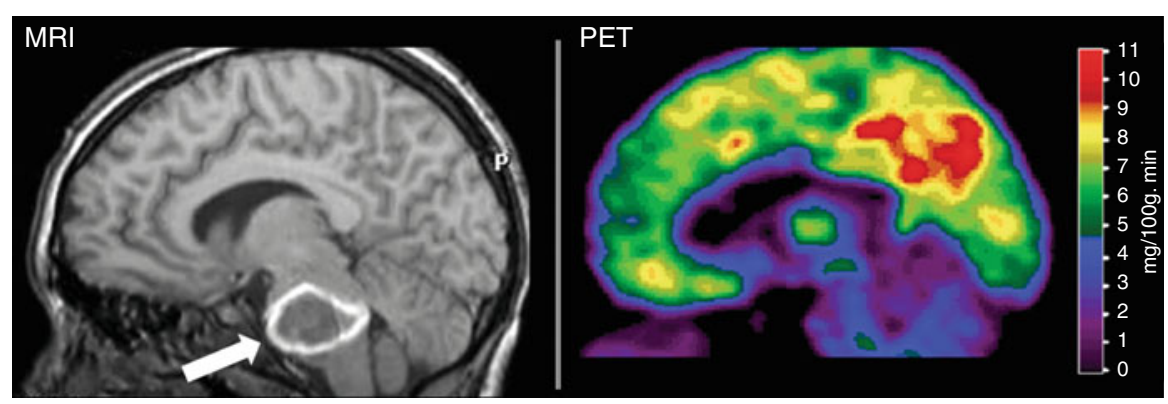

Fig. 7 Left: magnetic resonance image (MRI) showing a massive hemorrhage in the brain stem (arrow) causing a complete locked-in syndrome in a 13-year-old girl. Right: positron emission tomography (PET) scan illustrating intact brain function. The color scale shows the amount of glucose metabolized per $100 \mathrm{~g}$ of brain tissue per minute

Jean-Dominique Bauby, aged 43 and editor in chief of the fashion magazine "Elle," had a brain stem stroke. He emerged from a coma several weeks later to find himself in a locked-in syndrome only able to move his left eyelid. Bauby wanted to show the world that this disease, which impedes movement and speech, does not prevent patients from living. He has proven it in an extraordinary book in which he composed each passage mentally and then dictated it, letter by letter, to an assistant who painstakingly recited a frequency-ordered alphabet until Bauby chose a letter by blinking his eye once to signify "yes." His book The diving bell and the butterfly became a best seller only weeks after his death due to infection in 1997. Bauby created an Association for Locked-In Syndrome (www.alis-asso.fr) aimed to help patients with this condition and their families. Since its creation, they have registered over 500 locked-in patients in France.

Unless the physician is familiar with the signs and symptoms of the locked-in syndrome, the diagnosis may be missed, and the patient may erroneously be considered as being unconscious. Classically, structural brain imaging (X-ray CT or magnetic resonance imaging, MRI) shows isolated lesions of the brain stem that should caution the physician. However, studies have shown that in up to half of the cases, the first to realize the patient was conscious was the family and not the medical team. Most distressingly, the time elapsed between the brain insult and the diagnosis on average seems 2 months. The presence of a relatively normal and reactive electroencephalographic (EEG) rhythm in a patient that appears to be unconscious should alert to the diagnosis, but abnormal and unreactive EEG patterns can also be observed. So-called active cognitive event-related potentials have shown their utility to document consciousness in total locked-in syndrome. Figure 8 illustrates this method in a 21-year-old woman who failed to show any motor sign of consciousness up to 49 days after a basilar artery thrombosis. The patient was presented sequences of names containing the patient's own name and other names and was instructed to count her own name or to count another target name. A specific P3 wave could be recorded for the voluntarily counted names only, demonstrating the presence of command following and hence of consciousness. Two weeks later, the patient recovered some clinical signs of consciousness and later could communicate using a computer controlled by small finger movements. 


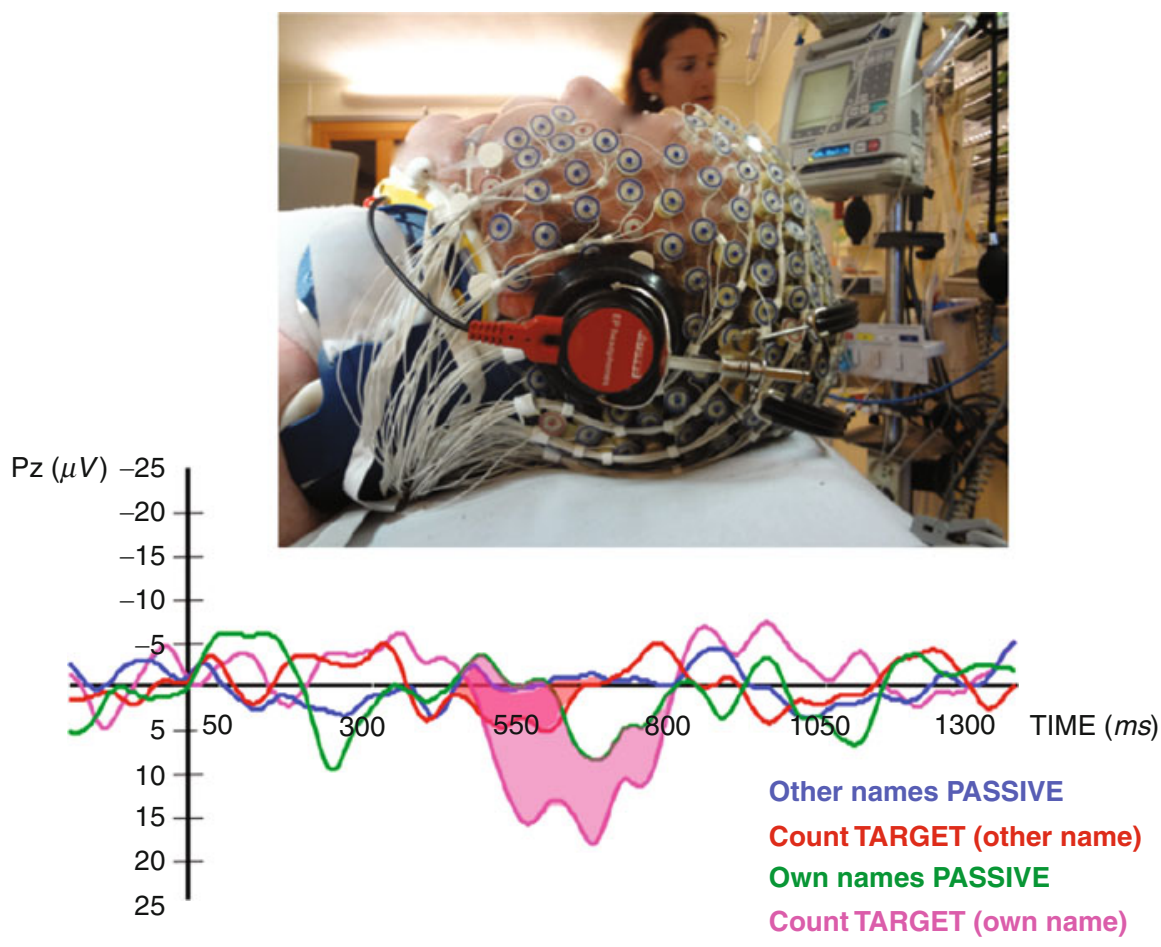

Fig. 8 "Active" event-related potentials showing command following ("count a name") and hence total locked-in syndrome. The increase in amplitude of the "P3 wave" (shown in pink) demonstrates that the clinically comatose patient understood and performed the task and, therefore, must be conscious

Positron emission tomography (PET) scanning has shown significantly higher global brain metabolism in the brains of patients in a locked-in syndrome compared to patients in coma or vegetative/unresponsive syndromes. No cortical area showed lower metabolism in acute and chronic locked-in syndrome patients when compared to age-matched healthy controls (Fig. 7). Conversely, hyperactivity was observed in bilateral amygdala of acute, but not chronic, locked-in syndrome patients. The absence of metabolic signs of reduced function in any area of the gray matter reemphasizes the fact that locked-in syndrome patients suffer from a pure motor deefferentation and recover an entirely intact intellectual capacity. Amygdala activation is known to be related to negative emotions such as fear and anxiety. It is difficult to make judgments about patient's thoughts and feelings when they awake from their coma in a motionless shell. However, it can be assumed that the increased activity in the amygdala, in the absence of decreased neural activity in any cortical region, relates to the terrifying situation of an intact awareness in a sensitive being, experiencing stress, anguish, and frustration, locked in an immobile body. Health-care workers should adapt their bedside behavior and consider pharmacological anxiolytic therapy of locked-in patients, taking into account the intense emotional state they go through. 
To allow measurements of intellectual functions in classical locked-in syndrome, adapted neuropsychological testing batteries based on eye-coded communication have been developed. In cases of pure brain stem lesions, patients will recover intact cognition, but additional cortical injuries can lead to associated cognitive deficits. For those not dealing with these patients on a daily basis, it is surprising to see how chronic locked-in syndrome patients, with the help of family and friends, can have essential social interaction and lead meaningful lives.

In the acute phase, mortality is high, but after the intensive care period, and given appropriate medical and nursing care, life expectancy can be many decades. Despite the classically encountered persisting serious motor deficit, most patients do recover some distal control of fingers and toe movements, sometimes allowing a functional use of a digital switch. The vast majority of patients remain dependent of others for activities of daily living. Given appropriate rehabilitation, most will recover some head or limb movement (although remaining wheelchair bound), and about half will be able to eat without gastrostomy, breath without tracheotomy, and recover some speech production (although limited to single comprehensible words).

Current eye-controlled computer-based communication technology is changing the lives of patients with locked-in syndrome (see video for example). Instead of passively responding to the requests of others, this technology allows patients to control their environment (lights, appliances, etc.), initiate conversations using a word processor, or access the Internet. Unfortunately, the cost of these devices is substantial and not routinely paid for by third parties. The preeminent physicist Stephen Hawking, author of A Brief History of Time, almost completely paralyzed due motor neuron disease, communicates using one finger and a computerized voice synthesizer. His continuing brilliant productivity despite the failure to move or speak illustrates that locked-in patients can be productive members of the society.

\section{Videos}

- Communication via fMRI, http://www.ulg.ac.be/cms/c_566156/steven-laureys

- Showing signs of consciousness using high-density EEG, http://www.ulg.ac.be/ cms/c_889273/a-new-door-on-consciousness

- Studying memories and near-death experiences, http://www.ulg.ac.be/cms/c 716080/near-death-experiences

A locked-in person moves a cursor on screen by eye movements. An infrared camera mounted below the monitor observes one of the user's eyes; an image processing software continually analyzes the video image of the eye and determines where he is looking on the screen. The patient looks at a virtual keyboard that is displayed on the monitor and uses his eye as a computer mouse. To "click," he looks at the key for a specified period of time (typically a fraction of a second) or blinks. An array of menu keys allow the user to control his environment, use a speech synthesizer, browse the World Wide Web, or send electronic mail independently (video used with kind permission from the patient). 


\section{“Vegetative" Wakeful Unresponsiveness}

In the 1970s, patients who awakened from coma (meaning they open their eyes spontaneously or after stimulation) but remained without communication or behavioral sign of consciousness were coined to be in a "vegetative state" (previously called "apallic syndrome" or "coma vigil") (Table 3). "Vegetative" was chosen to refer to the preserved vegetative (autonomous) nervous functioning in these patients (e.g., sleep-wake rhythm, respiration, digestion, and thermoregulation). The term "persistent" was added to denote that the condition remained for more than 1 month after insult. In 1994, a retrospective study of all published cases permitted to propose temporal boundaries for irreversibility of this syndrome, hence proposing the term "permanent vegetative state" (unfortunately, persistent and permanent vegetative state share the common abbreviation of "PVS," leading to unwarranted confusion). It is to these cases that end-of-life issues, of withholding and withdrawal of lifesustaining treatment (i.e., artificial hydration and nutrition), are related, as we will discuss later on.

Over the last three decades, some clinicians felt uncomfortable when referring to patients as "vegetative." Therefore, the term "unresponsive wakefulness syndrome" was recently proposed as a more neutral and descriptive term. The reasons for referring to these patients as "unresponsive" rather than "vegetative" are multiple. The word "vegetative" has an unintended albeit persistent negative connotation. The Oxford English dictionary defines "vegetative" as "an organic body capable of growth and development but devoid of sensation and thought" and "to vegetate" as to "live a merely physical life devoid of intellectual activity or social intercourse." The notion of a vegetative nervous system dates to the 1800 s when the nervous system was divided into animalic (i.e., related to sensory perception and voluntary motor responses) and vegetative parts (i.e., assuring nutritional functions). Unfortunately, for many laypersons, it has a pejorative undertone and may incorrectly refer to these patients as being "vegetable-like."

Some books written by patients erroneously considered as "vegetative" illustrate this point. A striking example is "Look Up for Yes" written by Julia Tavalaro (1997) who had a subarachnoid hemorrhage when she was 32 years old. She remained in a coma for 7 months and gradually woke up to find herself in a New York state chronic

Table 3 Diagnostic criteria for the "vegetative" state, as published by the US Multi-Society Task Force

No evidence of awareness of self or environment and an inability to interact with others

No evidence of sustained, reproducible, purposeful, or voluntary behavioral responses to visual, auditory, tactile, or noxious stimuli

No evidence of language comprehension or expression

Intermittent wakefulness manifested by the presence of sleep-wake cycles

Sufficiently preserved hypothalamic and brain stem autonomic functions to permit survival with medical and nursing care

Bowel and bladder incontinence

Variably preserved cranial-nerve and spinal reflexes 
care facility. There, she was known as "the vegetable," and it was not until 6 years later that her family identified a voluntary "attempt to smile." Later, using a letter board, she related the turmoil of her terrible years in captivity and could cheek-control her wheelchair around the hospital. Another poignant testimony is Only the Eyes Say Yes (1997) by Philippe Vigand. He had a vertebral artery dissection when he was 32 years old and remained in a coma for 2 months. He and his wife write that at first, doctors believed he was "a vegetable and was treated as such." His wife eventually realized that he was voluntarily blinking his eyes but had difficulties convincing the treating physicians. After many months of hospital care, he was brought home where an infrared camera attached to a computer enabled him to write his book. A recent Google search with "Terri Schiavo" and "vegetable" returned 410,000 hits and "Eluana Englaro" and "vegetable" gave 31,700 hits (performed April 25, 2011). A number of authors and social, political, and religious groups have hence felt the need to emphasize these patients' rights to be fully regarded as human beings.

In addition, "vegetative state" for many physicians implies "cortical death" and persistency from the moment of diagnosis. This already started when the New York Times (August 5, 1968) announced the Harvard criteria for brain death. In the accompanying editorial, it read: "As old as medicine is the question of what to do about the human vegetable... Sometimes these living corpses have survived for years... It is such cases, as well as the need for organs to be transplanted that the Harvard faculty committee had in mind in urging that death be redefined as irreversible coma." The case of Terri Schiavo also illustrates this point, as commentators have inaccurately referred to her condition as "brain death" or "neocortical death."

In the "vegetative" state, the brain stem is relatively spared, whereas the gray and/or white matter of both cerebral hemispheres are widely and severely damaged. Overall cortical metabolism of vegetative patients is $40-50 \%$ of normal values. Characteristic is a relative sparing of metabolism in the brain stem "activating reticular formation," hypothalamus, and basal forebrain. The functional preservation of these structures allows for their preserved arousal and autonomic functions. The other hallmark is a systematic impairment of metabolism in the polymodal associative cortices (bilateral prefrontal regions, Broca's area, parietotemporal and posterior parietal areas, and precuneus). These regions are known to be important in various functions such as attention, memory, and language. It has long been controversial whether the observed metabolic impairment in this large frontoparietal cortical network reflects an irreversible structural neuronal loss or functional and potentially reversible damage. However, in the rare cases where "vegetative" patients recover awareness, PET shows a functional recovery of metabolism in these same cortical regions. Moreover, the resumption of long-range functional connectivity between these associative cortices and between some of these and the intralaminar thalamic nuclei parallels the restoration of their functional integrity. The cellular mechanisms which underlie this functional normalization remain putative: axonal sprouting, neurite outgrowth, and cell division (known to occur predominantly in associative cortices in normal primates) have been proposed candidate processes. The challenge is now to identify the conditions in which, and the mechanisms by which, some vegetative patients may recover consciousness. 
Patients with disorders of consciousness are not uniformly hopeless, and increasing evidence from clinical and neuroimaging studies has shown that clinicians need to be cautious about making strong claims concerning allegedly "vegetative" patients' consciousness. Clinical practice shows that once stamped with the diagnosis "vegetative state," it frequently is difficult to change the label, and the first signs of recovery of consciousness are too often missed. Terry Wallis, who made the headlines when starting to speak after being considered as "vegetative" for 19 years posttrauma, well illustrates this point. Subsequent analysis of his medical files showed he actually was minimally conscious for all those years (albeit lacking proper rehabilitation).

\section{Minimally Conscious State}

Jin contingent relation to relevant environmental stimuli (such as reaching for objects that demonstrates a clear relationship between object location and direction of reach, touching or holding objects in a manner that accommodates the size and shape of the object, appropriate smiling or crying in response to the visual content of emotional, but not to neutral topics or stimuli). Future outcome studies should assess possible differences in prognosis, and neuroimaging studies could demonstrate the predicted differences in cognitive capacities and underlying functional neuroanatomy between these proposed subclasses.

Because criteria for the minimally conscious state have only recently been introduced, there are still few functional imaging studies of patients in this condition. Overall cerebral metabolism is decreased to values slightly higher but comparable to those observed in the "vegetative state." Metabolic activity in the medial parietal cortex (precuneus) and adjacent posterior cingulate cortex seems to best differentiate minimally conscious from "vegetative" patients. Interestingly, these areas are among the most active brain regions in conscious waking and are among the least active regions in altered states of consciousness such as general anesthesia, sleep, hypnotic state, dementia, and Wernicke-Korsakoff's or postanoxic amnesia. It has been suggested that this richly connected multimodal posteromedial associative area is a critical hub in the neural network subserving human internal awareness (Fig. 9). Simple auditory stimulation has been shown to induce a more widespread activation in minimally conscious than in "vegetative" patients. In the former, activation encompassed not only primary but also higher-order associative areas, suggesting a more elaborate level of processing. Moreover, corticocortical functional connectivity is more efficient in the MCS, compared to the VS, between auditory cortex and the frontoparietal "global neuronal workspace" considered critical in awareness, as will be discussed in the next section. Such findings encourage ongoing developments of neuromodulatory and cognitive revalidation therapeutic strategies in MCS patients.

In response to natural language stimuli (e.g., meaningful sentences), fMRI activation patterns of MCS patients exhibiting command following were examined by Schiff et al. during presentation of forward and backward narratives read in a familiar 


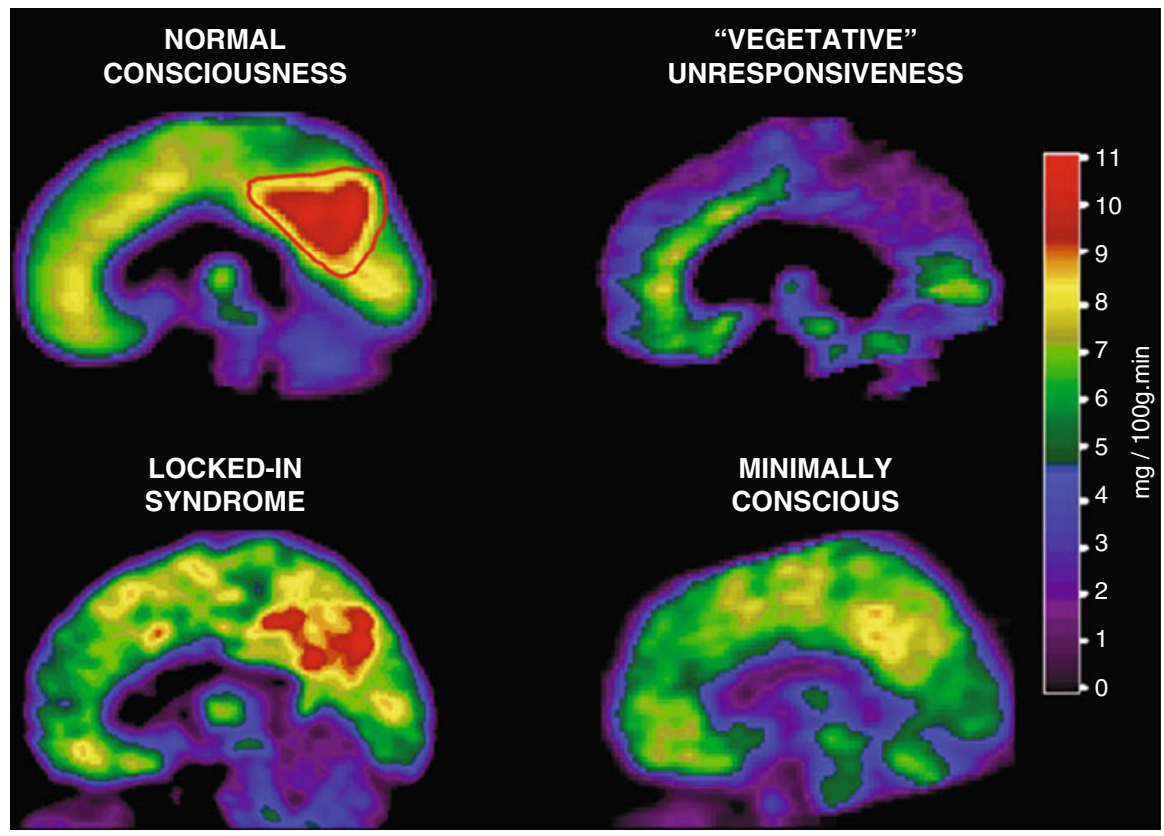

Fig. 9 In normal conscious waking, the medial posterior cortex (encompassing the precuneus and adjacent posterior cingulate cortex, delineated by a red line) is the metabolically most active region of the brain; in waking "vegetative"/unresponsive patients, this same area (delineated by a blue line) is the metabolically least active region. In the locked-in syndrome, no supratentorial brain region shows significant decreases in metabolism. In the minimally conscious state, the precuneus and posterior cingulate cortex shows an intermediate metabolism, higher than in vegetative patients, but lower than in conscious controls

voice and containing personally meaningful content. Components of the cortical language networks showed selective activation compared to baseline conditions. Presentation of the narratives time reversed (played backward) that shared most of the physical properties of the sounds activated the same networks as forward narratives in the normal controls subject, but failed to activate the networks in the MCS patients. These findings correlate with low resting metabolic activity and suggest that a residual capacity to activate large integrative networks may remain in some MCS patients. Preservation of large-scale networks in MCS patients may underlie rare instances of late recoveries of verbal fluency in such patients.

It is important to stress that the clinical diagnosis of these patients' (minimal) consciousness is based on the assessment of motor responsiveness. As will be discussed at the end of this chapter, functional neuroimaging (e.g., PET and functional MRI) and cognitive evoked potential studies have offered the possibility to measure directly and noninvasively severely brain-damaged patients' brain activity at rest and during external activation. More recently, these techniques have been further developed, aiming to detect "neural" (motor-independent) proof of command 
following and communication. Using fMRI, it was shown that some (exceptional) clinically "vegetative" patients could perform mental imagery tasks. When these patients were asked to imagine performing sport (e.g., tennis), robust activation could be recorded in motor areas. Other instructions such as "imagine moving around in your house" resulted in activation elsewhere in the brain (i.e., parahippocampal activation for spatial navigation tasks). These specific activations patterns were not different from those observed in healthy volunteers.

In a next step, scientists have employed this technique to establish fMRI-based communication. Here, patients are asked to answer autobiographical questions by doing motor imagery to answer "yes" and mental spatial navigation to answer "no" (Fig. 10). Note that many (minimally conscious) patients or classical locked-in patients fail to show reliable fMRI communication, illustrating the major limitations regarding the sensitivity of these techniques. Concurrently, cheaper and portable techniques using event-related potential or electromyography "active" paradigms are being developed to detect possible signs of command following not assessable by clinical behavioral examination.

The medical community at present has no diagnostic category for patients showing only signs of consciousness or communication on paraclinical fMRI or evoked potential studies. In the presence of increasingly "hard" neurophysiologic markers of consciousness, the burden of proof for establishing consciousness in severely brain-damaged patients no longer exclusively lies in behavioral assessment. Clearly, patients who can "play tennis" and "imagine walking in their house" or use these complex mental imagery tasks to accurately communicate cannot be considered "vegetative" or "minimally conscious." Some have proposed to call this

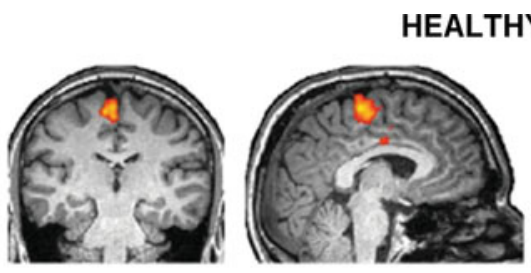

Answers «YES »

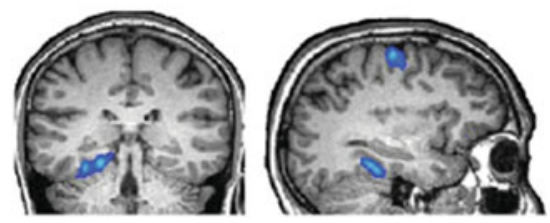

Answers « NO »

« VEGETATIVE STATE »
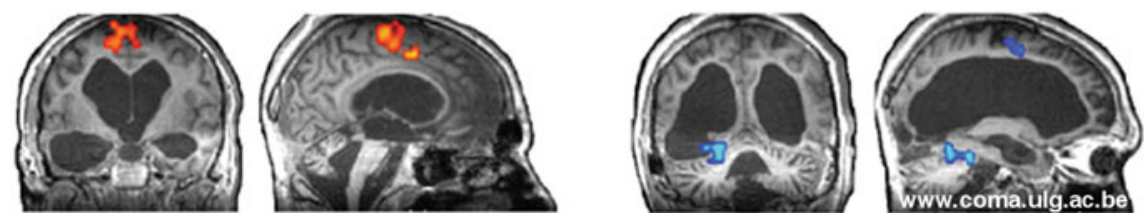

Fig. 10 Reliable fMRI-based communication in severely brain-damaged patients unable to show any motor-pathway-dependent communication. To answer "yes," a motor imagery task, activating motor areas (in orange), is performed. To answer "no," a spatial navigation task, activating another set of areas encompassing the parahippocampal regions (in blue), is performed 
condition "functional locked-in syndrome," emphasizing the dissociation between their extreme behavioral motor dysfunction and the identified preserved higher cognitive functions, as shown by functional imaging techniques.

Figure 10 Reliable fMRI-based communication in severely brain-damaged patients unable to show any motor-pathway-dependent communication. To answer "yes," a motor imagery task, activating motor areas (in orange), is performed. To answer "no," a spatial navigation task, activating another set of areas encompassing the parahippocampal regions (in blue), is performed.

\section{Ethical Issues and End-of-Life Decisions}

Treating patients with acute or chronic disorders of consciousness remains a challenging exercise. The debate on the need to continue or stop "futile" treatment in hopeless acute comatose states was started in the 1970s and is now widely accepted in intensive care. Today, almost half of all deaths in critical care units follow a decision to withhold or withdraw therapy. We know that the chances of recovery from chronic "vegetative" state are close to zero 1 year after traumatic and 3 months after nontraumatic brain damage. In these cases of permanent "vegetative" state, treatment can be considered futile, and its withdrawal can be ethically justified, based on the principles of patient's autonomy, beneficence, and nonmaleficence. For minimally conscious patients, outcome is considered to be better, and at present, no time intervals for possible permanency of the condition have been proposed. No generally accepted standards for care have been proposed for patients in minimally conscious state. How should we best care for these patients with chronic disorders of consciousness? Can treatment withholding or withdrawing be justified in some cases? It is generally accepted that competent patients should consent to any treatment they receive and have the right to make choices regarding their bodies and lives. The primary factor determining the level of treatment for an incompetent patient should reflect that patient's personally expressed wishes in his or her situation. But, by definition, coma, "vegetative"/ unresponsive, and minimally conscious patients cannot communicate their wishes.

Regarding locked-in syndrome, the American Academy of Neurology has published a position statement concerning the management of conscious and legally competent patients with profound and permanent paralysis. The conclusion is that such patients have the right to make health-care decisions about themselves, including whether to accept or refuse life-sustaining therapy - either not start or stop it once started. Doctors caring for locked-in syndrome have "an ethical obligation to minimize subsequent suffering" and should help patients with pain and dyspnea, "even if these medications contribute... to respiratory depression, coma, or death." However, patients should first be fully informed about their condition and the treatment options, and patients' decision must be consistent over a period of time. The latter is clearly necessary to exclude the impulsive transient reactions of despair that are common in patients with severe illness.

What is it like to be minimally conscious? Can patients with severe brain damage experience suffering or satisfaction? What is their quality of life? Is the level and 
content of consciousness in these patients with such severely damaged brains in any ways comparable to our own? These questions are very hard to answer. Some philosophers might even argue that the subjective aspect of the mind will not ever be sufficiently accounted for by the objective methods of reductionistic science. However, following a more pragmatic approach, many scholars believe that scientific and technological advances will ultimately improve our understanding and management of patients suffering from severe disorders of consciousness.

\section{Pain}

Like consciousness, pain is a subjective first-person experience. Many patients with severe brain damage cannot communicate their feelings and possible pain perception. The behavioral assessment of motor or autonomic signs (i.e., heart rate, respiratory frequency, blood pressure, pupillary diameter, and skin conductance) has shown not to be reliable indicators of conscious perception of pain (e.g., see studies done in general anesthesia). Pain management in severely brain-damaged patients constitutes a clinical and ethical stake. A recent survey of European medical professionals showed that the majority thought that patients in a "vegetative state" feel pain. More research is needed to increase our understanding of residual sensation in severely brain-damaged patients and to propose evidence-based medical guidelines for the management of possible pain perception and suffering in these vulnerable patient populations.

Very few functional neuroimaging studies have studied brain processing linked to noxious stimulation in the "vegetative state." When comparing cerebral activation to high-intensity electrical stimulation of the median nerve at the wrist in 15 "vegetative" patients, a preserved and robust activation of the brain stem, thalamus, and primary somatosensory cortex was observed in each and every patient. However, this residual activation was like an island, disconnected from the rest of the "pain matrix" (including the anterior cingulate cortex considered critical in the affective and cognitive processing of pain) and the higher-order cortical network considered necessary for conscious processing. Another study used a similar methodology in seven "vegetative" patients and confirmed activation in primary somatosensory cortex but also - and surprisingly in secondary somatosensory, insular, and anterior cingulate cortices. In the minimally conscious state, results have been more clear-cut and showed that painful stimulation activated the thalamus, somatosensory, insular, frontoparietal, and anterior cingulate cortices. No area was less activated in the "minimally conscious" patients, as compared to the studied healthy controls. The observation of a fully activated cerebral "pain matrix" gave objective evidence of a potential pain perception capacity in these patients, stressing the idea that they need analgesic treatment (Fig. 11).

The pros and cons of the use of painkillers in the severely brain-damaged patients, unable to communicate possible perception of pain, are very complex. Systematic use of narcotic analgesics in disorders of consciousness could lead to sedation and subsequent underestimation of signs of consciousness. On the contrary, some patients might experience hyperalgesia, requiring more aggressive analgesic therapy. Much 


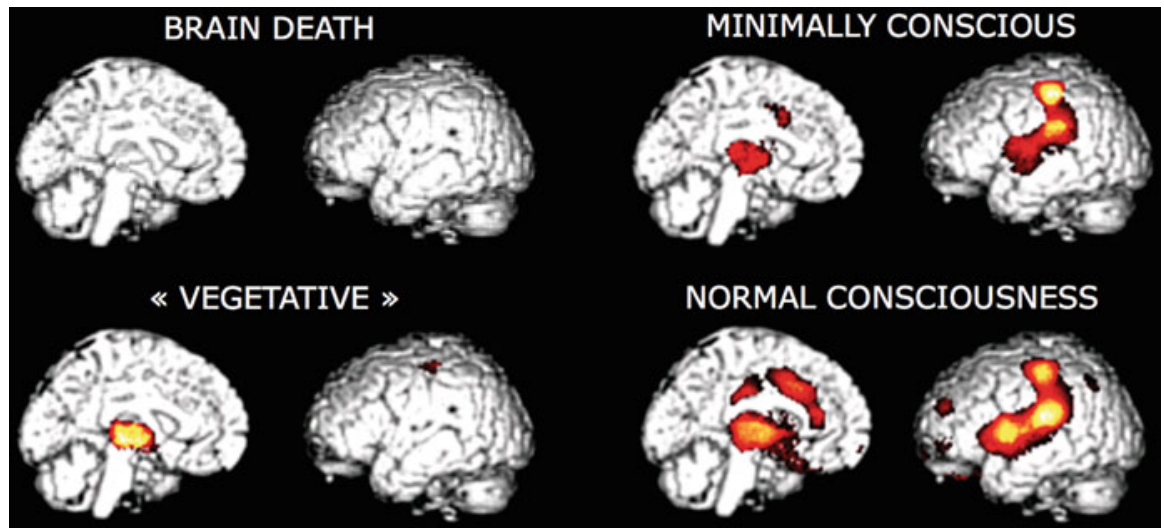

Fig. 11 Brain activation to noxious stimulation in brain death, "vegetative"/unresponsive wakefulness, and minimally conscious states, as compared to healthy volunteers. Note (a) the absence of activation in brain death; (b) the preserved but low-level subcortical and primary cortical activation in the vegetative state (the primary cortical activation was disconnected from the rest of the brain); and (c) the near-normal activation in the minimally conscious state

more research is needed in order to propose scientific-based guidelines. Such research, however, faces major ethical challenges. For some scholars, noxious stimuli cannot be applied to patients unable to give written informed consent. In disorders of consciousness, the exploration of behavioral responses to nociceptive stimuli (e.g., applying pressure to the fingernail bed with a pencil, applying pressure to the supraorbital ridge or jaw angle, pinching the trapezium, or rubbing the sternum) is a routine clinical procedure that is used to evaluate the state of consciousness. Reactivity to pain is part of widely used "consciousness scales" such as the Glasgow Coma Scale. Caring for severely brain-damaged patients represents such an immense humane, affective, and social problem that it warrants further research to better understand the underlying cerebral function of the severely damaged brain. Excluding the study of possible residual perception of pain from research protocols would not be ethically justifiable, but they need to be performed within well-defined ethical frameworks based on prudential ethic with respect to dissemination of new scientific methodology and technology, distinguishing investigational from clinical efforts and complementing neuroimaging studies with longitudinal epidemiologic inquiry into the natural history of disorders of consciousness. The ethical implications of these recent technological developments for public policy, emerging therapeutics, and diagnostic and prognostic assessment in these challenging patient populations will also need to be addressed.

\section{Quality of Life}

We cannot ask "vegetative" or minimally conscious patients about their selfperceived quality of life. However, we can ask locked-in patients who are also fully dependent of others for their daily life activities and survival. Recent studies 
have evaluated chronic locked-in patients' subjective well-being and the degree to which they are able to return to a "normal" life. Most chronic locked-in patients selfreport severe restrictions in community reintegration. Nevertheless, the majority seems to profess "good" subjective well-being (Fig. 12). This is in line with the notion that patients with severe disabilities may report a good quality of life despite being socially isolated or having major difficulties in activities of daily living. Interestingly, the longer patients are in a locked-in state, the higher self-reported quality of life seems to be. A minority of chronic locked-in patients declare to have a bad quality of life. Variables associated with unhappiness seem to be dissatisfaction with mobility in the community and anxiety. Studies have also shown that the presence of physical pain is correlated to the frequency of suicidal thoughts. These findings stress the importance (and current frequent inadequacy) of proper anxiety and pain management in chronic locked-in syndrome patients. The demand for euthanasia exists but seems uncommon. The principal clinical conditions for requests for euthanasia or physician-assisted death to be legally valid are "unbearable" suffering and irreversibility of the situation. Whereas the first condition may apply in some locked-in patients, irreversibility cannot be ascertained until, after the acute setting and rehabilitation, their subjective well-being has reached steady state, which may take as long as a year.

Results of studies on quality of life in locked-in syndrome may run contrary to many health-care professionals. Superficially involved for the short term when the patient is at his or her worst, clinicians may often tend to assume that these persons will die anyway or would choose to die if they only knew what the clinicians knew. As a result, debates about cost, daily management, quality of life, withdrawal or withholding of care, end-of-life decisions, and euthanasia often go on with prejudice and without any input from the conscious but mute and immobile patient. To "judge a book by its cover" is unfair. Clinicians should realize that quality of life often equates with social rather than physical interaction and that the will to live is strong when struck by an acute devastating disease.

Fig. 12 The majority of chronic locked-in syndrome patients self-report a good quality of life, as illustrated using Anamnestic

Comparative Self-Assessment scores

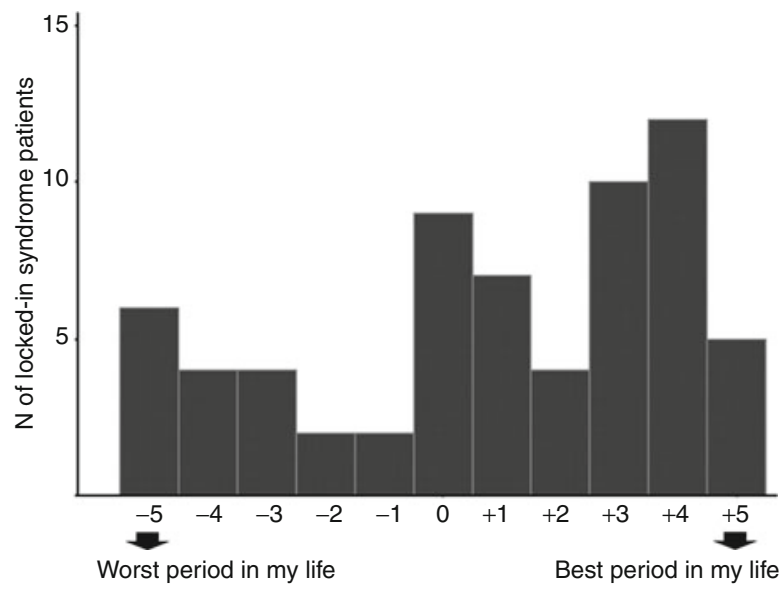




\section{Neural Signature of Consciousness}

In addition to its clinical and ethical importance, studying severely brain-damaged patients offers a still largely underestimated means to the study of human consciousness. The contrastive approach comparing brain activation in circumstances that do or do not give rise to consciousness in either of its two main senses of wakefulness and awareness is now widely applied in functional neuroimaging. In contrast to other unconscious states such as general anesthesia and sleep, where impairment in arousal cannot be disentangled from impairment in awareness, we are here offered a unique lesional approach enabling us to identify the neural correlates of (un) awareness. The discussed neuroimaging and electrophysiology studies are illuminating the relationships between conscious awareness and (1) global brain function, (2) regional brain function, (3) changes in functional connectivity, and (4) primary versus associative cortical activation in response to external stimulation.

For a long time, it is known that arousal is maintained by a diffuse system of upper brain stem and thalamic neurons (called "reticular activating system") and its connections to the cerebral hemispheres. Therefore, depression of either brain stem or global hemispherical function may cause reduced wakefulness. Therefore, as we will see, measuring brain stem reflexes is a key to the assessment of coma and the patient's functional integrity of the brain stem's arousal systems. The neural correlate of awareness, however, is only recently better understood. As we will see, it can be regarded as an emergent property of the functional integrity of widespread "higherorder" frontoparietal feedback loops with "lower-level" sensory systems.

Is awareness lost when overall cortical activity falls below a certain threshold? In the vegetative/unresponsive state, global metabolic activity decreases to about $50 \%$ of normal levels - similar to what is observed in anesthesia and deep sleep. However, in patients who recover, global metabolic rates for glucose metabolism do not necessarily show substantial recovery. Hence, the relationship between global levels of brain function and awareness is not absolute. Consciousness is not an emergent property of diffuse global brain function or connectivity. Some areas are more important than others for its emergence.

Using voxel-based statistical analyses in the study of severe brain damage, a systematic dysfunction in a wide frontoparietal network was observed when patients might have recovered arousal but lacked conscious awareness. This network encompasses polymodal associative cortices of both the convexity (bilateral, dorsolateral, prefrontal, and posterior parietal areas) and midline (mesiofrontal/anterior cingulate and precuneal/posterior cingulate cortices). These results have been confirmed in other diseases where patients also are seemingly "wakeful" but only show reflex automatic behavior lacking "voluntary interaction with others" - such as absence and complex partial seizures and sleepwalking.

Current analyzing techniques now also permit to assess awareness-related changes in functional integration. Long-range corticocortical and corticothalamocortical "functional disconnections" could be identified in vegetative/unresponsive patients. Moreover, recovery of awareness after severe brain damage seems paralleled by a functional restoration of this corticothalamocortical connectivity 
(i.e., with nonspecific intralaminar thalamic nuclei). This understanding culminated in the use of deep brain stimulation therapy to improve awareness in traumatic braininjured minimally conscious patients.

Hemodynamic functional neuroimaging studies (fMRI and PET) using external (noxious or auditory) stimulation showed robust activation in primary and sensory "lower-level" areas which were however isolated and dissociated from the "higherorder" frontoparietal cortical network. The activation in primary cortices in awake but unaware patients confirms Francis Crick's early hypothesis (based on visual perception and monkey histological connectivity) that neural activity in primary cortices is necessary, but not sufficient for awareness. This leads to the understanding that cerebral sensory "slave systems" are important for shaping the content of perceptual awareness, but it is the functional integration in the frontoparietal neural workspace that appears critical for the emergence of conscious awareness.

It was also proposed that conscious awareness has two main dimensions: external awareness (i.e., perception coming via our senses) and internal awareness (i.e., stimulus-independent thought, mental imagery, or inner speech). fMRI studies seem to indicate that functional connectivity of the lateral frontoparietal workspace network is important for external awareness, while midline frontoparietal network connectivity related to internal awareness, known to be the most active "by default" in resting nonstimulated conditions (Fig. 13). Recently, evoked potential studies using dynamic causal modeling in severely brain-damaged patients showed that it is the top-down "backward" connections from the frontoparietal network which seem critical for awareness. Taken together, these studies show that consciousness can be regarded as an emergent property of the

Fig. 13 The frontoparietal "conscious network" can be divided in an external lateral network (in red) critical for external or sensory awareness and an internal midline network (in blue) critical for internal or self-awareness

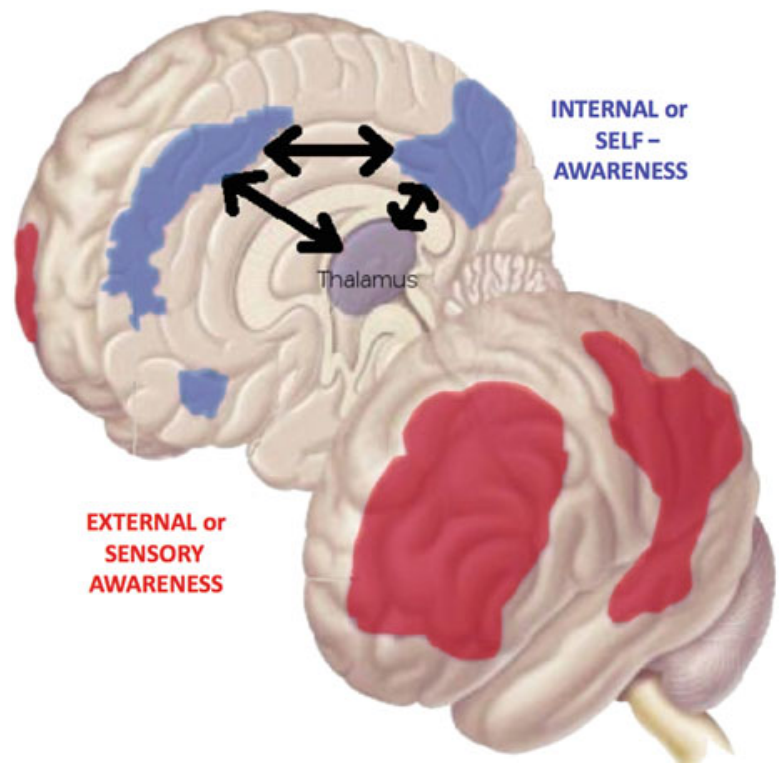


collective behavior of frontoparietal top-down functional connectivity. Of clinical importance, this knowledge now permits to improve the diagnosis of patients with disorders of consciousness, which, as we have seen, remains very challenging at the bedside. As discussed, current technology and knowledge now also permit to show command-specific changes in EEG or fMRI signals providing motorindependent evidence of conscious thoughts and in some cases even of communication.

\section{Outlook}

Some patients who awaken from their coma may fail to show any behavioral sign of awareness (i.e., they are considered to be "vegetative" or - as is preferred "unresponsive"), or they may remain unable to communicate, i.e., are in a minimally conscious state - now subdivided in MCS+ (i.e., showing more complex behaviors such as command following) and MCS- (i.e., showing only nonreflex movements). The clinical management of these disorders of consciousness remains very challenging, but technological advances in neuroimaging are now offering new ways to improve our diagnosis. It is an exciting time as the behaviorally defined gray zones between the different disorders of consciousness in the clinical spectrum following coma are being challenged by increasingly powerful imaging technology. For the first time, neurologists may encounter rare but existing cases of "functional locked-in syndrome," where only paraclinical tests permit to demonstrate the presence of higher cognitive function, inaccessible to our motor-dependent clinical evaluations.

In a not so far future, real-time fMRI-based communication or evoked potential brain-computer interfaces will be used to address important clinical and ethical questions such as feeling of pain and discomfort. These novel technological means will undoubtedly further improve the existing nosology and clinical care of these challenging patients with disorders of consciousness. Clearly, it is a thrilling time for the field of acute brain injury. The gray zones between the different disorders of consciousness in the clinical spectrum following coma are beginning to be better defined by the addition of powerful imaging methodology to bedside clinical assessment.

It is to be foreseen that in a yet to be discovered fraction of patients who are currently considered to be "vegetative" or clinically unresponsive, functional MRI, event-related potential, or electromyography techniques will reveal signs of consciousness that are unattainable by bedside clinical assessment. These novel technological means will further change the existing behaviorally defined boundaries between the various consciousness disorders. In a next step, these high-tech devices will permit some of these clinically "noncommunicative" patients to correspond their thoughts and wishes via nonmotor pathways. However, it should be stressed that these exciting developments are not yet reality. The first hurdle to be overcome before the methodologies discussed above can enter routine clinical practice relates to ethical concerns. Current ethical frameworks emphasize the need to achieve a balance between the protection of patients with disorders of consciousness and the ability to perform research that can lead to medical progress. Most of the advances in 
coma science discussed above are based on small cohort or even single-case studies. Only large-scale multicenter clinical trials will enable these research tools to find their way into evidence-based care for individuals with a disorder of consciousness following severe brain damage.

Acknowledgments SL is supported by the Belgian Funds for Scientific Research (FRS), James S. McDonnell Foundation, European Commission (Mindbridge, DISCOS, DECODER, and COST), Mind Science Foundation, University and University Hospital of Liège, and Belgian French-Speaking Community Concerted Research Action.

\section{References}

Boly M, Garrido MA, Gosseries O, Bruno MA, Boveroux P, Schnakers C, Massimini M, Litvak V, Laureys S, Friston K (2011) Preserved feedforward but impaired top-down processes in the vegetative state. Science 332:858-862

Bruno MA, Bernheim J, Ledoux D, Pellas F, Demertzi A, Laureys S (2011) A survey on selfassessed wellbeing in a cohort of chronic locked-in syndrome patients: happy majority, miserable minority. BMJ Open 1(1):e000039

Fins JJ, Illes J, Bernat JL, Hirsch J, Laureys S, Murphy E (2008) Neuroimaging and disorders of consciousness: envisioning an ethical research agenda. Am J Bioeth Neurosci 8:3-12

Giacino J, Hirsch J, Schiff N, Laureys S (2006) Functional neuroimaging applications for assessment and rehabilitation planning in patients with disorders of consciousness. Arch Phys Med Rehabil 87:67-76

Laureys S (2005) Death, unconsciousness and the brain. Nat Rev Neurosci 11:899-909

Laureys S (2007) Eyes open, brain shut: the vegetative state. Sci Am 4:32-37

Laureys S, Tononi G (2008) The neurology of consciousness. Academic, New York

Laureys S, Owen A, Schiff N (2004) Brain function in coma, vegetative state, and related disorders. Lancet Neurol 3:537-546

Laureys S, Pellas F, Van Eeckhout P, Ghorbel S, Schnakers C, Perrin F, Berré J, Faymonville ME, Pantke KH, Damas F, Lamy M, Moonen G, Goldman S (2005) The locked-in syndrome: what is it like to be conscious but paralyzed and voiceless. Prog Brain Res 150:495-511

Monti MM, Vanhaudenhuyse A, Coleman MR, Boly M, Pickard JD, Tshibanda JF, Owen AM, Laureys S (2010) Willful modulation of brain activity in disorders of consciousness. N Engl J Med 362:579-589

Owen AM, Coleman MR, Boly M, Davis MH, Laureys S, Pickard J (2006) Detecting awareness in the vegetative state. Science 313:1402

Owen AM, Schiff ND, Laureys S (2009) A new era of coma and consciousness science. Prog Brain Res 177:399-411

Posner JP, Saper CB, Schiff ND, Plum F (2007) Plum and posner's stupor and coma. Oxford University Press, Oxford/New York

Schiff ND, Giacino JT, Kalmar K, Victor JD, Baker K, Gerber M, Fritz B, Eisenberg B, Biondi T, O'Connor J, Kobylarz EJ, Farris S, Machado A, McCagg C, Plum F, Fins JJ, Rezai AR (2007) Behavioural improvements with thalamic stimulation after severe traumatic brain injury. Nature 448:600-603 